Surgery for

Congenital

Heart Disease

\title{
Comparison of extracardiac Fontan techniques: Pedicled pericardial tunnel versus conduit reconstruction
}

\author{
Ronald K. Woods, MD, PhD a \\ Umesh Dyamenahalli, $\mathrm{MD}^{\mathrm{a}}$ \\ Brian W. Duncan, MD ${ }^{\mathrm{b}}$ \\ Geoffrey L. Rosenthal, MD, PhD \\ Flavian M. Lupinetti, MD ${ }^{c}$
}

From the Divisions of Pediatric Cardiothoracic Surgery and Pediatric Cardiology, the University of Washington School of Medicine, Seattle, Wash, ${ }^{\mathrm{a}}$ the Cleveland Clinic, Cleveland, Ohio, 'b and Phoenix Children's Hospital, Phoenix, Ariz. ${ }^{c}$

Read at the Twenty-eighth Annual Meeting of The Western Thoracic Surgical Association, Big Sky, Mont, June 19-22, 2002.

Received for publication June 27, 2002; revisions requested Aug 1, 2002; revisions received Aug 23, 2002; accepted for publication Aug 28, 2002.

Address for reprints: Flavian M. Lupinetti, MD, 1144 E. McDowell Rd, Suite 204, Phoenix, AZ 85006 (E-mail: fmlupinetti@ hotmail.com).

J Thorac Cardiovasc Surg 2003;125:465-71 Copyright $\odot 2003$ by The American Association for Thoracic Surgery

0022-5223/2003\$30.00+0

doi: $10.1067 / \mathrm{mtc} .2003 .153$
Objective: This study was designed to determine whether either of 2 alternative methods of extracardiac Fontan reconstruction provides superior results.

Methods: We reviewed 58 consecutive Fontan procedures performed between 1995 and 2001 with a pedicled pericardial tunnel (group P, $\mathrm{n}=21$ ) or an extracardiac conduit of polytetrafluoroethylene or allograft aorta (group $\mathrm{C}, \mathrm{n}=37$ ). Operations were performed with cardiopulmonary bypass at $32^{\circ} \mathrm{C}$; an aortic crossclamp was applied in only 6 patients. All group P patients and 33 (89\%) group C patients received fenestrations.

Results: The groups were similar in terms of age, weight, anatomy, and preoperative hemodynamics. There were 3 hospital deaths (5\%; 70\% confidence limit, 2\%-30\%), all in group C. Median durations of mechanical ventilation (group P, 1 day; group C, 1 day), intensive care unit stay (group P, 3 days; group C, 3 days), chest tube drainage (group P, 8 days; group C, 7 days), and hospitalization (group P, 10 days; group C, 9 days) were not significantly different. There were no late deaths. All patients received warfarin sodium, and there were no late strokes. Before the Fontan procedure, 1 patient in group $\mathrm{P}$ and 3 patients in group $\mathrm{C}$ required pacemaker implants. Of the 51 surviving patients in sinus rhythm before the Fontan procedure, only 1 patient in group $\mathrm{C}$ subsequently required a pacemaker.

Conclusions: Extracardiac Fontan procedures with either a pericardial baffle or conduit are associated with low operative mortality and low risks of arrhythmia and late thromboembolic complication.

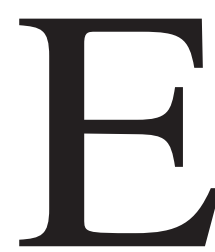

fforts to improve the circulatory function of patients with singleventricle physiology have focused on characteristics such as flow dynamics, thrombogenicity, atrial incisions and suture lines, growth potential, and ease of surgical reconstruction, resulting in several modifications of the original Fontan procedure. ${ }^{1-3}$ The widely used extracardiac total cavopulmonary connection with a synthetic conduit or homograft can be constructed without cardiac ischemia or large atrial incisions and has performed well in several large series. ${ }^{2-6}$ The use of pedicled pericardium for the extracardiac connection is a more recent modification with additional potential advantages. Gundry and colleagues ${ }^{7}$ observed good flow char- 
acteristics and growth of the connection in a series of 19 patients. Growth potential is theoretically a significant advantage because a large proportion of total cavopulmonary connection procedures are now performed in children before age 2 to 3 years. Pedicled pericardium might also have a lesser tendency for infectious and thromboembolic complications. However, experience with pedicled pericardial reconstruction is limited and has not been compared with reconstruction with synthetic conduits or allograft vessels in a contemporary series.

This study reviews an experience with extracardiac Fontan repairs initiated in 1995. Pedicled pericardium was, from the beginning, adopted as the technique of choice. Prosthetic or allograft conduits also were used, usually because of unfavorable anatomy or lack of pericardium. The 2 types of reconstruction used in this series were compared with respect to both immediate and longer-term outcomes.

\section{Patients and Methods}

\section{Patient Population}

The medical records of all patients undergoing a Fontan procedure from January 1, 1995, through December 31, 2001, at a single children's hospital were reviewed. Before 1995, all Fontan procedures performed within this institution were some variety of intracardiac operations. Beginning in 1995, the extracardiac Fontan procedure was used exclusively. Demographic information, cardiac anatomy, pre-Fontan operative and nonoperative treatment, operative details, and postoperative outcomes were recorded. Patients, families, cardiologists, and primary care physicians were contacted when necessary to acquire current follow-up information. Permission for performance of this study was obtained from the institutional review board.

\section{Operative Techniques}

Extracardiac Fontan reconstruction with a pedicled pericardial baffle, as described by Gundry and associates, ${ }^{7}$ was considered the preferred operative approach. This method was undertaken for all patients whose congenital anatomy permitted this procedure and whose pericardium was adequate for the purpose. The operative technique included the use of cardiopulmonary bypass with moderate hypothermia $\left(32^{\circ} \mathrm{C}\right)$. Cannulation of the aorta and the superior vena cava was performed in standard fashion. On bypass, the inferior vena cava was clamped at its junction with the right atrium, and the anterolateral wall of the inferior vena cava was incised. The proximal junction of the cava and the atrium was oversewn with a 5-0 polypropylene suture, closing the atrium and allowing removal of the clamp. The inferior vena cava was not cannulated; rather return of inferior vena caval blood to the bypass circuit was accomplished with cardiotomy suction. The pericardium on the systemic venous side was mobilized, with care taken not to damage the phrenic nerve. The pericardium was incised at its superior extent in a perpendicular direction toward the superior vena cava and at its inferior extent toward the inferior vena cava. The posteromedial aspect of the pericardium remained attached, allowing the pericardium to assume a hemicylindrical shape, with the atrial free wall as its floor. The inferior margin of the pericar- dium was sewn to the edge of the partially divided inferior vena cava, and this suture line was continued for a short distance onto the atrial free wall. The distal pulmonary arteries were then controlled, and a pulmonary arteriotomy was performed. The posterior wall of the opened pulmonary artery was sewn to the dome of the atrium. The superior margin of the pericardial baffle was sewn to the anterior wall of the pulmonary artery, and this suture line was also continued onto the atrial free wall. The suture lines from the superior and inferior ends were then continued toward each other. An effort was made to place all sutures on the atrial free wall in a superficial fashion and to avoid any full-thickness suture lines. Just before completion of the atrial suture line, a 5-mm fenestration between the pericardial baffle and the lumen of the atrium was created with a punch. There was no attempt made to adjust the size of the fenestration on the basis of patient size or other considerations.

When the pericardium was inadequate to permit a baffle construction or when congenital anomalies required a more complicated routing of inferior vena caval blood, an extracardiac conduit was used for the Fontan repair. As for the baffle reconstruction, cardiopulmonary bypass with moderate hypothermia was used. The inferior vena cava was divided, and an end-to-end anastomosis was performed with a conduit. Initial patients received an allograft aortic conduit; subsequent patients received a conduit of expanded polytetrafluoroethylene (PTFE).* The other end of the conduit was anastomosed to the pulmonary artery. The caliber of the PTFE tube (or allograft) was 16 to $18 \mathrm{~mm}$ on the basis of the measured caliber of the inferior vena cava. A fenestration, if used, was performed by creating parallel 5- to 6-mm holes in the PTFE tube and the atrial free wall and anastomosing them with 5-0 PTFE sutures.

If patients required an additional intracardiac procedure, perfusate temperatures were reduced to $27^{\circ} \mathrm{C}$, and cardioplegic arrest was performed before the Fontan repair. In all other cases not requiring intracardiac procedures, the aorta was not crossclamped, and the myocardium was perfused throughout the operation. This required particular attention to avoid air emboli.

Routine monitoring included a superior vena caval catheter, usually placed percutaneously after induction of anesthesia, and an atrial catheter placed intraoperatively.

\section{Statistical Analysis}

Mean values and SDs were calculated for continuous variables. Comparisons were made between patients who underwent the Fontan procedure with the extracardiac pericardial baffle and those who received an extracardiac conduit. All analyses were performed with StatView statistical software (Abacus Concepts, Berkeley, Calif).

\section{Results}

\section{Operative Details and Patient Characteristics}

During the 7-year period from 1995 to 2001, there were 58 Fontan procedures performed. Of these, a pedicled pericardial baffle was performed in 21 patients, and an extracardiac conduit was performed in 37 patients. Fenestrations were performed at the time of the Fontan procedure in all patients

*Gore-Tex; registered trademark of W. L. Gore \& Associates, Inc, Flagstaff, Ariz. 


\section{TABLE 1. Congenital cardiac anatomy}

\begin{tabular}{lr}
\hline Pericardial baffle $(\mathrm{n}=21)$ & 7 \\
Hypoplastic left-heart syndrome & 5 \\
Tricuspid atresia & 4 \\
Pulmonary atresia-intact septum & 2 \\
Unbalanced atrioventricular septal defect & 2 \\
Double-inlet left ventricle & 1 \\
Double-outlet right ventricle & \\
Extracardiac conduit ( $\mathrm{n}=37)$ & 11 \\
Hypoplastic left-heart syndrome & 6 \\
Unbalanced atrioventricular septal defect & 6 \\
Double-inlet left ventricle & 5 \\
Tricuspid atresia & 5 \\
Pulmonary atresia-intact septum & 3 \\
Double-outlet right ventricle & 1 \\
Ebstein malformation &
\end{tabular}

undergoing a pericardial baffle and in $32(86 \%)$ patients having an extracardiac conduit. Conduits used were aortic allografts in 4 patients and PTFE conduits in 33 patients. In addition to the Fontan procedure, other procedures were performed in 2 patients who underwent a pericardial baffle (1 repair of an insufficient atrioventricular valve and 1 atrial septectomy) and 6 patients who received a conduit (2 repairs of insufficient atrioventricular valves, 2 atrial septectomies, 1 resection for subaortic stenosis, and 1 translocation of an anomalous coronary artery). Cardiac arrest times for these 8 patients ranged from 11 to 66 minutes (mean, $35 \pm 21$ minutes).

Patient ages in the pericardial baffle group (mean, $38 \pm$ 14 months; median, 37 months) and in the conduit group (mean, $46 \pm 28$ months; median, 40 months) were not significantly different. Patient weights in the pericardial group (mean, $13.2 \pm 1.8 \mathrm{~kg}$; median, $12.9 \mathrm{~kg}$ ) and in the conduit group (mean, $15.4 \pm 6.0 \mathrm{~kg}$; median, $14.0 \mathrm{~kg}$ ) also were not significantly different.

Congenital cardiac defects are displayed in Table 1. The largest single group of cardiac diagnoses was hypoplastic left heart syndrome, which occurred in almost a third of the overall patient population. In the pericardial baffle group the dominant ventricle exhibited left ventricular morphology in 13 patients and right ventricular morphology in 8 patients. Among the extracardiac conduit group, the dominant ventricle was of left ventricular morphology in 20 patients and right ventricular morphology in 17 patients. First-stage palliations are shown in Table 2. Consistent with the anatomic diagnoses, systemic-pulmonary artery shunts and the Norwood operation were the most common initial operations. Eleven patients, however, did not have a typical first-stage operation because of balanced systemic and pulmonary flow.

All patients, regardless of initial palliation or lack thereof, underwent a bidirectional Glenn procedure at age 4 months to 7 years (mean, $13.1 \pm 13.6$ months; median, 8.7

\section{TABLE 2. Stage 1 operative procedures}

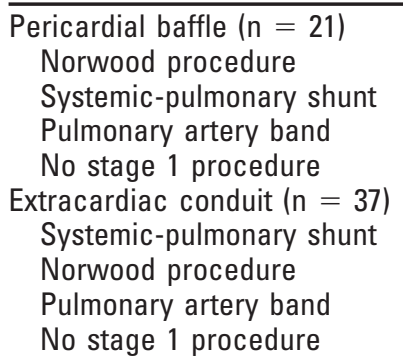

months). The bidirectional Glenn procedure was accompanied by additional operative procedures in 9 patients: 3 unifocalizations of discontinuous pulmonary arteries, 2 atrial septectomies, 1 Damus-Kaye-Stansel procedure, 1 pulmonary arterioplasty, 1 tricuspid valvuloplasty, and 1 pacemaker insertion.

\section{Perioperative Morbidity and Mortality}

There were 3 (5\%; 70\% confidence limit, 2\%-30\%) inhospital deaths. All deaths occurred in the conduit group, and all of these patients had a fenestration. One death was due to an air embolus occurring during cardiopulmonary bypass. A second death occurred in a patient with severe heart failure before the Fontan procedure. The patient was successfully extubated and managed for a period of time but died on postoperative day 35 with persistent heart failure. The third death occurred in a patient with increased pulmonary vascular resistance who died on postoperative day 6 . In retrospect, this patient might have survived had the Fontan repair been taken down. Thus in no case did the use of the conduit, as opposed to the pericardial baffle, appear to have influenced mortality. The patients who died did not have preoperative cardiac catheterization measurements that might have predicted a more difficult course. In each case the measured pressures and calculated resistances were within the usual range of acceptability for this operation. One additional patient with increased pulmonary resistance who underwent a PTFE conduit repair underwent takedown of the repair on postoperative day 1 and survived.

Seven additional surviving patients had important complications. One patient with a nonfenestrated PTFE conduit had low cardiac output and ascites but responded successfully to operative revision and creation of a fenestration. Four patients ( 2 with aortic allografts, 1 with a PTFE conduit, and 1 with a pericardial baffle) were found to have had mild strokes, as indicated by a computed tomographic scan. The ages of the strokes were not always clear, and it was not possible to determine whether these events occurred in the immediate perioperative period or had preceded the operations by some length of time. All of these patients had good recovery, with minimal or no sequelae. Two patients 
( 1 with a pericardial baffle and 1 with a PTFE conduit) had mediastinitis that required operative debridement and repair of the chest wall without revision of the cardiac reconstruction. Despite the use of cardiotomy suction for the inferior vena caval return, hemolysis did not appear to be an important problem.

\section{Hospital Course}

For all patients who were hemodynamically stable, efforts were made to extubate at the earliest possible time postoperatively. The median duration of mechanical ventilation in hospital survivors was 1 day in each group. The median duration of intensive care unit hospitalization among survivors was 3 days in each group. The median duration of chest tube drainage was 7 days in the pericardial baffle group and 8 days in the conduit group. The median duration of total hospital stay was 9 days in each group. None of these differences is statistically significant.

\section{Follow-up and Late Results}

Information regarding the late status of patients was obtained from medical records, office records of cardiologists and primary care physicians, and personal contact with patients and families. Follow-up during calendar year 2001 was accomplished for 49 (89\%) of 55 patients surviving hospitalization. Mean duration of follow-up was 23 months. Overall follow-up was $88.6 \%$ complete. There were no late deaths. Three patients underwent late reoperations: 1 patient with a pericardial baffle received a pleurectomy for recurrent pleural effusions, 1 patient with a conduit received a pericardial window for recurrent pericardial effusions, and 1 patient with a conduit received a diaphragm plication for diaphragm paralysis.

All patients were started on warfarin sodium (Coumadin) beginning on postoperative day 2 , with a goal of achieving an international normalized ratio of 1.5 to 2.0. Heparin and antiplatelet agents were not used. The duration of continuing warfarin sodium was left to the discretion of the patient's personal cardiologist; however, it was recommended that warfarin sodium be continued indefinitely when compliance could be predicted and there were no contraindications. There was no intention of selectively using warfarin therapy on the basis of the type of Fontan modification, presence or absence of a fenestration, cardiac rhythm, or other factors.

Patients were otherwise free of any other late complications sometimes associated with the Fontan procedure. Specifically, no patients had protein-losing enteropathy, and there were no late strokes or other clinical events consistent with cerebral emboli, no other systemic embolic events, no hemorrhagic complications, and no pulmonary venous complications.

\section{Electrocardiographic Results}

Electrocardiographic results were based on 12-lead electrocardiograms and selective use of Holter monitors, as clinically indicated. Pacemakers had been implanted in 5 patients before undergoing the Fontan procedure or concomitantly with the Fontan procedure. Of the 19 patients who underwent pericardial baffle repair and who were in sinus rhythm before the Fontan procedure, all remain in sinus rhythm at an acceptable rate at the time of their latest electrocardiogram 2 to 73 months postoperatively (mean, 15 months). Among the 31 patients who were in sinus rhythm at the time of their extracardiac conduit Fontan procedure and survived operation, 30 remain in sinus rhythm 2 to 69 months postoperatively (mean, 26 months). The remaining patient with conduits required a pacemaker for sinus bradycardia.

\section{Patency of Fenestrations}

Among the 21 patients who had a pericardial baffle, all of whom had fenestrations placed intraoperatively, 3 fenestrations were closed with catheter-delivered closure devices because of excessive right-to-left shunting, 4 closed spontaneously, and 14 remain patent, as determined by means of echocardiography. Among the 29 surviving patients who had fenestrations with an extracardiac conduit Fontan procedure, 1 underwent transcatheter closure, 1 underwent surgical closure, 12 underwent spontaneous closure, and 15 remain patent.

\section{Discussion}

Pericardium has been used in a variety of cardiovascular reconstructive procedures. Its use in constructing a cavopulmonary connection was first described in 1992 by Hvass and associates, ${ }^{8}$ who constructed a tube of pericardium to connect the inferior vena cava to the pulmonary artery. Other reports describe Fontan constructions with only autologous tissue by using native atrial wall and septum or unusually located inferior cavae.9-10 In 1997, Gundry and colleagues ${ }^{7}$ reported their series of extracardiac pericardial reconstructions, in which pericardium was attached to the lateral wall of the right atrium or the interatrial groove to connect the inferior vena cava to the pulmonary artery. Of 19 patients, there were no operative deaths or subsequent complications of thromboembolism or arrhythmia. Moreover, follow-up studies confirmed growth of the pathway.

The current series includes patients who had extracardiac Fontan procedures on the basis of the approach of Gundry and colleagues, ${ }^{7}$ as well as a contemporaneous group undergoing repair with extracardiac synthetic conduits. A comparison of these alternative techniques within a single institution by surgeons whose approach is otherwise consistent has not been previously reported. An evaluation of routine clinical parameters revealed that both types of re- 
constructions were associated with low mortality, short length of stay, acceptable duration of pleural drainage, and few longer-term complications, with follow-up extending beyond 6 years in both groups. Longer-term follow-up might reveal advantages related to the growth potential of the pericardial reconstruction. In the present study there is not documentation of growth. In part this relates to the difficulty in retrospective analysis of the patients' echocardiographic studies. In the absence of uniform techniques, personnel, and measurement strategies, consistent measurements might be lacking, and data on the basis of these sites are of dubious validity.

Pathway construction with pedicled pericardium can be somewhat more technically demanding. In a high percentage of patients in this series, the pericardium was unavailable or of inadequate integrity for the reconstruction. In no patient in this series was an insufficient quantity of pericardium augmented with contralateral pericardium or prosthetic tissue. Had that been done, it might have been possible to reduce the frequency of conduit repair. Also technically challenging is the complicated anatomic relationship commonly observed in patients with heterotaxy syndrome. In these patients the remoteness of the inferior vena cava can increase the challenges of a pericardial baffle. All heterotaxic patients in this series had a conduit repair. Regardless of the type of extracardiac approach, reconstruction can be performed without cardiac ischemia and with bypass times that are similar to those of other techniques. Yet another technical issue is injury to the phrenic nerve. This problem is sometimes encountered in other modifications of the Fontan procedure as well, but this was observed in only one patient in this series.

The relative merits of the flow properties of these alternative pathways are unknown. It is possible that the pliability of the pericardial baffle degrades efficiency of energy transfer along the pathway. However, this pliability also allows transmission of intrathoracic pressure and respiratory variation, which might ultimately provide greater enhancement of flow. ${ }^{7,11}$ Patients in this series underwent echocardiographic and angiographic evaluations that have revealed low velocity flow without significant turbulence through either the pericardial baffle or the extracardiac conduit. Clinical parameters, such as oxygen saturation, cardiac output, pleural fluid drainage, ascites, and enteropathy, although quite indirect measures of hemodynamic performance, did not differ significantly between the 2 types of reconstructions in this series.

The almost uniform use of a fenestration in this series is one difference from the series of Gundry and colleagues. ${ }^{7}$ The use of the fenestration was favored because it appeared to reduce the perioperative instability in patients undergoing Fontan procedures with only a minimal reduction in arterial saturation. The fenestration also appeared to benefit some patients in the longer term by helping to maintain a more adequate cardiac output. The routine use of a fenestration has resulted in a small number of patients who have required later closure of this fenestration because of excessive desaturation. In most cases the fenestration closes spontaneously or remains patent without adverse effects. The patency rate of fenestrations and the need for fenestration closure were similar in the pericardial and conduit groups.

All patients in this series, regardless of the type of material used for the reconstruction, were treated with warfarin because of the prothrombotic tendency of the Fontan circulation. ${ }^{12,13}$ It might be speculated that a pericardial baffle is at lower risk of thrombus formation than a prosthetic conduit. However, other variables, such as caliber of the connection, volume of flow, pulmonary artery capacitance, and cardiac function, might be stronger influences on the thromobotic potential of the Fontan connection. The small number of patients and thromboembolic events in this series preclude a meaningful conclusion as to the superiority of one approach over the other.

Five children in this series ultimately received pacemakers: 3 were implanted before the Fontan procedure, and 1 was implanted in each of the 2 groups after the Fontan procedure. Although atrial incisions, and perhaps suture lines, might promote arrhythmias, most authors recognize the cumulative effects of multiple procedures, dissections in the area of the sinoatrial node, and hemodyamic factors in the genesis of arrhythmias. ${ }^{14-16}$ The current data indicate that both types of Fontan procedure have a low frequency of arrhythmias within the time frame of this review. The long lag time between Fontan procedures and the subsequent development of arrhythmias, however, indicates that caution should be exercised in drawing conclusions regarding the ultimate arrhythmogenicity of these techniques.

Limitations of the present study include the relatively small numbers of patients in the 2 groups and the lack of randomization. The more complicated anatomy in the conduit group, taken as a whole, probably biases the results against this technique. In addition, it is recognized that many of the complications of the Fontan operation and its modifications occur many years after the procedure. Protein-losing enteropathy and arrhythmias are perhaps the 2 most dangerous late sequelae that rarely occur within the first 5 years after the Fontan procedure.

From these data, it is concluded that either pedicled pericardium or synthetic conduits can be used for constructing the extracardiac Fontan repair, with no significant differences in clinical performance, mortality, or complications. It will be important to maintain longer follow-up periods to determine whether the growth potential of pedicled pericardium is realized and whether either approach over additional years affects rhythm disturbances, thromboembolic events, and length and quality of life. 


\section{References}

1. Gentles TL, Mayer JE, Gauvreau K, Newburger JW, Lock JE, Kupferschmid JP, et al. Fontan operation in 500 consecutive patients: factors influencing early and late outcome. J Thorac Cardiovasc Surg. 1997; 114:376-91.

2. Pearl JM, Laks H, Stein DG, Drinkwater DC, George BL, Williams R. Total cavopulmonary anastamosis versus conventional modified Fontan procedure. Ann Thorac Surg. 1991;52:189-96.

3. Knott-Craig JC, Danielson GK, Schaff HV, Puga FJ, Weaver AL, Driscoll DD. The modified Fontan operation. An analysis of risk factors for early postoperative death or takedown in 702 consecutive patients from one institution. J Thorac Cardiovasc Surg. 1995;109: 1237-43.

4. Petrossian E, Reddy VM, McElhinney DB, Akkersdijk GP, Moore P, Parry AJ, et al. Early results of the extracardiac Fontan operation. J Thorac Cardiovasc Surg. 1999;117:688-96.

5. Giannico S, Corno A, Marino B, Cicini MP, Gagliardi MG, Amodeo A, et al. Total extracardiac right heart bypass. Circulation. 1992; 86(suppl II):II110-7.

6. Shirai LK, Rosenthal DN, Reitz B, Robbins RC, Dubin AM. Arrythmias and thromboembolic complications after the extracardiac fontan operation. J Thorac Cardiovasc Surg. 1998;115:499-505.

7. Gundry SR, Razzouk AJ, del Rio MJ, Shirali G, Bailey LL. The optimal Fontan connection: a growing extracardiac lateral tunnel with pedicled pericardium. J Thorac Cardiovasc Surg. 1997;114:552-9.

8. Hvass U, Pansard Y, Bohm G, Depoix JP, Enguerrand D, Worms AM. Bicaval pulmonary connection in tricuspid atresia using an extracardiac tube of autologous pediculated pericardium to bridge inferior vena cava. Eur J Cardiothorac Surg. 1992;6:49-51.

9. Hashimoto K, Kurosawa H, Tanaka K, Yamagishi M, Koyanagi K, Ishii $\mathrm{S}$, et al. Total cavopulmonary connection without the use of prosthetic material: technical considerations and hemodynamic consequences. J Thorac Cardiovasc Surg. 1995;110:625-32.

10. van Son JAM, Reddy VM, Hanley FL. Extracardiac modification of the Fontan operation without use of prosthetic material. J Thorac Cardiovasc Surg. 1995;110:1766-8.

11. Fogel MA, Weinburg PM, Hoydu A, Hubbard A, Rychik J, Jacobs M, et al. The nature of flow in the systemic venous pathway measured by magnetic resonance blood tagging in patients having the Fontan operation. J Thorac Cardiovasc Surg. 1997;114:1032-41.

12. Jahangiri M, Kreutzer J, Zurakowski D, Bacha E, Jonas RA. Evaluation of hemostatic and coagulation factor abnormalities in patients undergoing the Fontan operation. J Thorac Cardiovasc Surg. 2000; 120:778-82.

13. Balling G, Vogt M, Kaemmerer H, Eicken A, Meisner H, Hess H. Intracardiac thrombus formation after the Fontan operation. $J$ Thorac Cardiovasc Surg. 2000;119:745-52.

14. Cohen MI, Bridges ND, Gaynor JW, Hoffman TM, Wernovsky G, Vetter VL, et al. Modifications of the cavopulmonary anastamosis do not eliminate early sinus node dysfunction. J Thorac Cardiovasc Surg. 2000;120:891-901.

15. Kavey RW, Gaum WE, Byrum CJ, Smith FC, Kveselis DA. Loss of sinus rhythm after total cavopulmonary connection. Circulation. 1995; 92:304-8.

16. Manning PB, Mayer JE Jr, Wernovsky G, Fishberger SB, Walsh EP. Staged operation to Fontan increases the incidence of sinoatrial node dysfunction. J Thorac Cardiovasc Surg. 1996;111:833-40.

\section{Discussion}

Dr Steven R. Gundry (Palm Springs, Calif). I congratulate the authors on their study comparing the pericardial lateral tunnel that bears my name with an extracardiac prosthetic lateral tunnel. It is said that an inventor of an operation can hopefully achieve great results, but it is only until these results are achieved by others that any new operation should be taken seriously. I thank you for your article from the bottom of my pericardium.

I will not summarize the authors' results because they have been shown so well today. Rather, I will concentrate on those areas where the authors' techniques differ from our experience at Loma Linda and now at the International Heart Institute of Palm Springs.

The authors state that although the pericardial lateral tunnel is their preferred technique, it could only be used in $35 \%$ of their patients because of an absence of adequate pericardium or the presence of complex anatomy. In our experience we have not had to resort to prosthetic extracardiac devices, even in cases of complex anatomy and complex tunneling, often using the pericardial tunnel beneath the free left ventricle and complex anatomy or supplementing inadequate pericardium from one side with pericardium taken from the other side. Our late results continue to support this approach. Although you mention reasons in your article, why did you abandon your preferred approach in the majority of patients for a prosthetic conduit?

Dr Woods. This is the teacher talking to the ultimate author. If your experience with doing that is showing good results, then I think Dr Lupinetti or I would consider trying it. Part of that low instance reflects the patient population in some degree also in that if you are having a triply staged pathway, then you are going to be more in the chest and with lesser qualities of pericardium for your final stage. The ultimate thing that guided the operations in these cases was brevity and simplicity. If the procedure was going to entail a more detailed pericardial reconstructive technique, then it was abandoned.

Dr Gundry. Good answer. You used fenestrations in all of your patients, a technique that we abandoned in 1992. I note that you did have a $7 \%$ stroke rate and that $10 \%$ of your patients have required subsequent intervention for closure of the fenestration, both catheter based and surgical, and that all your patients had to take warfarin. What do you think are the reasons why you would continue to use these techniques that support fenestrations in light of the significant morbidity associated with fenestrations?

Dr Woods. We have not documented any specific fenestrationrelated morbidity. The use of warfarin was purely elective at the outset of the series on the basis of work by others. In terms of the strokes that occurred, 3 of those actually occurred in children in whom PTFE was used for reconstruction. Only 1 occurred in a child with a fenestration, and therefore it is hard for us to attribute that to fenestration-passage thromboembolic material. In our experience it is anecdotal but it seems that-and in discussing this with the interventional cardiologist before I left Seattle who does our fenestration closure- the children seem to be getting out of the hospital more quickly with less effusion and less time to resumption of normal function, but it is certainly a matter of choice involved, as well as objectivity.

Dr Gundry. This actually brings me to my final question. Your time to discharge is a median of 9 days. That does not seem cost effective. It seems to be related to chest tube drainage time. Beginning in 1994, as you might know, we began sending patients home with Blake drains in place and actually teaching the mothers to empty these. My partner, Leonard Bailey, currently holds the record for a first-postoperative-day discharge using this technique, while I pale to his series with a second-postoperative-day discharge in the majority of my cases. Have you considered using Blake drains to speed earlier discharge?

Dr Woods. That has been done in a limited fashion in the more recent months. Of course, much depends on your referral population and your comfort level. With referrals that are across state 
lines, we are a little less comfortable doing that, but with local referrals we do occasionally use early discharge.

Dr Gregory Fontana (Los Angeles, Calif). I would like to compliment the authors as well on a fantastic series. We continue the search for the holy grail along the Fontan pathway. Certainly, we are not near the end.

I am concerned about any sort of atrial incisions. In many clinical series the most significant long-term morbidity after a Fontan procedure is atrial arrhythmias. In laboratory studies various atrial incisions or suture lines have been shown to augment the substrate of atrial arrhythmias. Clearly, there are some theories of the pedicled periventricular tunnel to the prosthetic external conduit; however, I am concerned about the potential risk for development of atrial arrhythmias caused by the pericardial pedicle.

Dr Woods. I fully share your concerns. We await further follow-up. As you know, the studies that have looked at that have shown that it is a multifactorial process. It does not just necessarily relate to incisions or suture lines in the area but also hemodynamic factors, the number of times you go back in. Peter Manning published a study in the last couple of years demonstrating that the causes are multifactorial. I guess partial thickness in suture lines will have some role, but that is not an objective view either. We share your concerns, but there are not enough objective data proving the contrary, and therefore it seems feasible to at least monitor these patients long term by electrocardiographic followup.

Dr Fontana. What is your indication for the pedicle pericardial technique? Is it for patients with unusual anatomy? Is it for the smaller patient in whom you are hoping for some growth potential? Or is it on a case-by-case basis?

Dr Woods. As a general statement, each case is approached with a preference to using pericardium. Of course, it is going to be modified on an individual basis, and I think that in children who have had maybe multiple re-entries for complications or something like that, where their pericardium is subsequently of lesser quality, there might be some selection bias involved with that. However, in general, if the pericardium is there, if it can be dissected and mobilized and it is not pitted with multiple areas of thinning, it will be used whether the child is 4 or 6 or $12 \mathrm{~kg}$.

Dr Fontana. Are you saying that the basis for this technique is to achieve total autologous surfaces?

Dr Woods. Yes.

Dr Hillel Laks (Los Angeles, Calif). I also would like to congratulate you on the excellent results. The proposed advantage of the extracardiac conduit is that you have a rigid tube and laminar flow. With the pericardial tube, I would assume that you have a structure that is less tubular and more irregular. Have any studies, such as magnetic resonance angiography through 3-dimen- sional reconstruction, been done to look at the shape of these tubes and how much turbulence you have? Also, with the issue of follow-up echocardiograms in all of these patients, do you have any evidence of areas of stenosis at the inferior vena caval orifice with long-term follow-up, and what is the evidence that growth occurs in your patients with the longest follow-up? Last, the proposed advantage of lateral tunnel and natural tissue is of course the lack of the use of anticoagulants. Why have you continued to use anticoagulants long term in the patients receiving pericardial tubes and have you had any experience with not using anticoagulants in that group?

Dr Woods. Paranoia is pretty much the last factor. We can discuss this with Dr Lupinetti, but the prothrombotic or altered thrombotic state of these children is a known entity, and whether you are using synthetic or native tissue, they are still existing in an altered coagulation state. I think it is for that reason, combined with a little bit of paranoia, that anticoagulation is still done. It might be that in the future this will be stopped, but it is not something that we have discussed at this point. Therefore, we might be able to appreciate that advantage in the future.

In terms of the follow-up echocardiographic analysis, the actual inferior portion in our series - and I would be interested to hear what Dr Gundry's series has shown-is actually quite patent and, if anything, capacious. It is at the superior end, where the vein comes underneath, that there is, if anything, relative appearance of narrowing, although there are no gradients that we have been able to pick up. We have used a stent in one child in our series, I believe, since analysis of these data who had some narrowing in that area.

In terms of flow analysis, that is something that is in an institutional review board that is still in the process of being submitted by using magnetic resonance to analyze flow in these pathways. As you can imagine, locating children after a post-third stage reconstruction and getting them to come back in is taking some time. The only 2 magnetic resonance studies that I am aware of that have looked at flow in Fontan pathways have been the ones from Morgan in 1998 and Fogel in 1997. They both used magnetic resonance. One used blood tagging, but they were not specifically analyzing these types of conduits. In Dr Gundry's article, respiratory variation was discussed in terms of its effects on the flow, and I personally share your concern in terms of the loss of the mechanical advantage, the hydrodynamics of the stiff rigid tube. However, according to Gundry's article, some augmentation is provided by respiratory variation that is easily transmitted to the wall of that tunnel and might offset some of the mechanical energy losses that you get with a nonrigid structure. Beyond that, I do not have a good answer. 